\title{
Predicting Pathologic Response of Esophageal Cancer to Neoadjuvant Chemotherapy: The Implications of Metabolic Nodal Response for Personalized Therapy
}

\author{
John M. Findlay ${ }^{1,2}$, Kevin M. Bradley ${ }^{3}$, Lai Mun Wang ${ }^{2,4}$, James M. Franklin ${ }^{3}$, Eugene J. Teoh ${ }^{3}$, Fergus V. Gleeson ${ }^{3}$, \\ Nicholas D. Maynard ${ }^{1}$, Richard S. Gillies ${ }^{1}$, and Mark R. Middleton ${ }^{2,5}$ \\ ${ }^{1}$ Oxford OesophagoGastric Centre, Churchill Hospital, Oxford, United Kingdom; ${ }^{2}$ NIHR Oxford Biomedical Research Centre, \\ Churchill Hospital, Oxford, United Kingdom; ${ }^{3}$ Department of Nuclear Medicine, Churchill Hospital, Oxford, United Kingdom; \\ ${ }^{4}$ Department of Pathology, John Radcliffe Hospital, Oxford, United Kingdom; and ${ }^{5}$ Department of Oncology, University of Oxford, \\ Old Road Campus Research Building, Oxford, United Kingdom
}

\begin{abstract}
Only a minority of esophageal cancers demonstrates a pathologic tumor response (pTR) to neoadjuvant chemotherapy (NAC). ${ }^{18} \mathrm{~F}-\mathrm{FDG}$ $\mathrm{PET} / \mathrm{CT}$ is often used for restaging after NAC and to assess response. Increasingly, it is used during therapy to identify unresponsive tumors and predict pTR, using avidity of the primary tumor alone. However, definitions of such metabolic tumor response (mTR) vary. We aimed to comprehensively reevaluate metabolic response assessment using accepted parameters, as well as novel concepts of metabolic nodal stage $(\mathrm{mN})$ and metabolic nodal response $(\mathrm{mNR})$. Methods: This was a single-center retrospective U.K. cohort study. All patients with esophageal cancer staged before NAC with PET/CT and after with CT or PET/CT and undergoing resection from 2006 to 2014 were identified. pTR was defined as Mandard tumor regression grade 1-3; imaging parameters included metrics of tumor avidity $\left(\mathrm{SUV}_{\mathrm{max} / \mathrm{mean} / \text { peak }}\right.$ ), composites of avidity and volume (including metabolic tumor volume), nodal $\mathrm{SUV}_{\max }$, and our new concepts of $\mathrm{mN}$ stage and mNR. Results: Eighty-two (27.2\%) of 301 patients demonstrated pTR. No pre-NAC PET parameters predicted pTR. In 220 patients restaged by PET/CT, the optimal tumor $\triangle \mathrm{SUV}_{\text {max }}$ threshold was a $77.8 \%$ reduction. This was as sensitive as the current PERCIST $30 \%$ reduction, but more specific with a higher negative predictive value $(P<0.001)$. $\Delta \mathrm{SUV}_{\max }$ and $\Delta$ length independently predicted $\mathrm{PTR}$, and composite avidity/spatial metrics outperformed avidity alone. Although both $\mathrm{mTR}$ and $\mathrm{mNR}$ were associated with pTR, in 82 patients with ${ }^{18} \mathrm{~F}-\mathrm{FDG}$-avid nodes before NAC we observed mNR in $10(12.2 \%)$ not demonstrating mTR. Conclusion: Current definitions of metabolic response are suboptimal and too simplistic. Composite avidity/volume measures improve prediction. $\mathrm{mNR}$ may further improve response assessment, by specifically assessing metastatic tumor subpopulations, likely responsible for disease relapse, and should be urgently assessed when considering aborting therapy on the basis of mTR alone.
\end{abstract}

Key Words: esophageal cancer; neoadjuvant therapy; positronemission tomography; precision oncology

J Nucl Med 2017; 58:266-275

DOI: 10.2967/jnumed.116.176313

Received Mar. 29, 2016; revision accepted Jul. 13, 2016.

For correspondence or reprints contact: John M. Findlay, Oxford OesophagoGastric Centre, Churchill Hospital, Oxford, U.K. OX3 7LJ.

E-mail: john.findlay@oncology.ox.ac.uk

Published online Sep. 15, 2016.

COPYRIGHT (C) 2017 by the Society of Nuclear Medicine and Molecular Imaging.
$\mathbf{I}_{\mathrm{n}}$ treatment of esophageal cancer is neoadjuvant chemotherapy (NAC) or chemoradiotherapy (NACR) followed by surgery $(1,2)$. Both confer important survival benefits (3); however, up to $60 \%$ of tumors show either minimal or no pathologic response (pTR) to NAC (4,5), and a similarly poor response is seen in $30 \%-40 \%$ after NACR (6). For these patients such, in retrospect, futile therapy delays surgery, potentially allowing disease progression and a worse prognosis (7). The ability to predict pTR at the outset would therefore be invaluable, as it would allow personalized therapy, with neoadjuvant therapy being omitted or changed to alternative therapy in those patients unlikely to benefit.

The evidence for the predictive value of baseline molecular markers and PET is insufficiently robust to justify major treatment changes $(8,9)$. Interval assessment of response during therapy is, therefore, the next best option for personalizing therapy. Interval tumor metabolic response (mTR) on PET predicts pTR, albeit imperfectly. A 35\% reduction in $\mathrm{SUV}_{\max }$ is most commonly used during therapy $(10,11)$ and formed the basis of the landmark MUNICON trial, wherein NAC was continued after a single cycle only in patients with a reduction in $\mathrm{SUV}_{\text {max }}$ greater than 35\% (12); the alternative PERCIST recommend a $30 \%$ reduction after NAC to define mTR (13). However, these thresholds may not be optimal: PERCIST is neither tumor- nor contextspecific, whereas the MUNICON threshold was derived from just 40 patients; furthermore, $\mathrm{SUV}_{\text {max }}$ provides no spatial information. More fundamentally, both assess only the primary tumor; the high rates of disease recurrence seen even in patients with pathologically responsive primary tumors suggests important unidentified factors, perhaps involving nodal or distant micrometastases-a recent report described tumor downstaging after NAC (a reduction from pretreatment clinical to posttreatment pathologic stage) to be strongly associated with survival (14).

With this in mind, we recently explored the novel concepts of ${ }^{18}$ F-FDG-avid nodal stage ( $\mathrm{mN}$ stage) and metabolic nodal response (mNR) and demonstrated major clinical implications for identifying disease progression during NAC, independent of primary tumor stage and response (15).

In this study, we aimed to reexamine comprehensively the utility of PET/CT in predicting pTR to NAC. First, we assessed the 
predictive ability of clinical, pathologic, and imaging factors available before NAC. Second, we aimed to define and compare optimal thresholds of mTR after NAC and assess, for the first time, to our knowledge, the novel concept of mNR. Third, we aimed to generate and validate predictive models that might have clinical utility.

\section{MATERIALS AND METHODS}

\section{Patients and Staging Protocol}

All patients who underwent potentially curative surgical resection of esophageal/gastroesophageal junctional cancer and were staged initially with CT and ${ }^{18} \mathrm{~F}$-FDG PET/CT were identified from a departmental database (May 2006 to November 2014) (16). This included all cell types. The study was approved by the institutional clinical governance department, and the need for written informed consent was waived. Patients were also staged with endoscopic ultrasound and laparoscopy for tumors extending below the diaphragm as previously described (16). Examinations were reported by a consultant upper gastrointestinal radiologist/gastroenterologist using the contemporary American Joint Committee on Cancer TNM staging manual (sixth (17) or seventh edition (18)).

\section{NAC}

NAC was considered for all patients with disease more advanced than T1N0. Patients with esophageal and gastroesophageal junction (GEJ) Siewert 1/2 tumors (19) received either cisplatin and 5-fluorouracil (5-FU; 2 cycles; $n=182$ ) (20); oxaliplatin and 5-FU ( 2 cycles; $n=$ 46) (21); epirubicin, cisplatin, and 5-FU (ECF; 3 cycles; $n=7$ ); epirubicin, cisplatin, and capecitabine (ECX; 3 or 4 cycles; $n=22$ ) (22); epirubicin, oxaliplatin, and capecitabine (EOX; 3 cycles; $n=$ 3 ); cisplatin and etoposide ( 2 cycles; $n=1$ ); or oxaliplatin and capecitabine ( 2 cycles; $n=1$ ). Patients with type 3 GEJ tumors received ECX/EOX/ECF (3 cycles). Some patients (distal esophageal/GEJ) received 3 cycles of ECX pre- and postoperatively with $(n=7)$ or without bevacizumab $(n=20)(23)$, or 3 cycles of ECF pre- and postoperatively $(n=12)(24)$.

\section{Restaging CT and PET/CT}

Patients were restaged 4-6 wk after NAC using CT before 2008 and $\mathrm{PET} / \mathrm{CT}$ afterward (although a small minority underwent CT because of clinical trial protocols) as previously described (16). ${ }^{18} \mathrm{~F}$-FDG PET/ CT was performed using 1 of 2 scanners. Before November 3, 2009, scans were obtained on a Discovery STE (GE Healthcare) $60 \mathrm{~min}$ after injection of $400 \mathrm{MBq}$ of ${ }^{18} \mathrm{~F}$-FDG. Images were reconstructed using a time-of-flight ordered-subset expectation maximization reconstruction algorithm (2 iterations, 20 subsets; field of view, $50 \mathrm{~cm}$; matrix, 128; voxel size, $3.9 \times 3.9 \times 3.3 \mathrm{~mm}$; 2D). After November 3, 2009, scans were obtained on a Discovery 690 (GE Healthcare) $90 \mathrm{~min}$ after injection of ${ }^{18} \mathrm{~F}-\mathrm{FDG}(4 \mathrm{MBq} / \mathrm{kg})$. Images were reconstructed using a time-of-flight ordered-subset expectation maximization reconstruction algorithm ( 2 iterations, 24 subsets; gaussian filter, $6.4 \mathrm{~mm}$; field of view, $50 \mathrm{~cm}$; matrix, 256; voxel size, $1.95 \times 1.95 \times 3.3 \mathrm{~mm}$; 3D). Examinations were independently reported by 2 dedicated PET/CT radiologists.

\section{Operations}

Surgery was typically performed within $2 \mathrm{wk}$ of the restaging scan. A minimum 2-field lymphadenectomy was performed as standard.

\section{Data and Variables}

Patient variables included age, sex, and American Society of Anesthesiologists grade (25); pretreatment tumor variables were cell type, grade (26), anatomic site, $\mathrm{T}$ (seventh edition), $\mathrm{N}$ stage (sixth edition because data were insufficient for conversion to the seventh), and whether the tumor was impassable at esophagogastroduodenoscopy. PET/CT variables are described below. NAC variables comprised a dual- or triple-agent regimen (due to large number of regimens and small patient groups) and time (d) from staging to restaging scan and scan to surgery to adjust for delays and number of cycles given. pTR was defined as Mandard Tumor Regression Grade (TRG) of 3 or less, after dedicated review by a consultant cellular pathologist (27). The Mandard TRG was used in preference to alternative TRGs, being the most frequently used TRG for esophageal cancer (28), with optimal prediction of survival $(29,30)$.

\section{PET/CT Variables}

Variables comprised primary tumor ${ }^{18} \mathrm{~F}-\mathrm{FDG}$ avidity $\left(\mathrm{SUV}_{\max }\right.$ and length [cm]), mN stage, $\mathrm{mNR}$, and $\mathrm{SUV}_{\max }$ of the most ${ }^{18} \mathrm{~F}-\mathrm{FDG}$-avid node. The development of $\mathrm{mN}$ stage and $\mathrm{mNR}$ have been described previously (15). $\mathrm{mN}$ stage (nodes visible discretely from the tumor, within a standard lymphadenectomy territory, with $\mathrm{SUV}_{\text {max }}>2.5$ or background mediastinal blood pool) comprised mN0 (0 avid nodes), $\mathrm{mN} 1$ (1-2 nodes), and $\mathrm{mN} 2$ (>2 nodes). mNR comprised complete metabolic response or partial metabolic response (reduction in $\mathrm{mN}$ or $\mathrm{SUV}_{\text {max }} \geq 30 \%$ ), stable metabolic disease (stable $\mathrm{mN}$ or reduction/ progression $\mathrm{SUV}_{\max }<30 \%$ ), or progressive metabolic disease (progression of $\mathrm{mN}$ or $\mathrm{SUV}_{\max } \geq 30 \%$ ).

For examinations using the second PET/CT scanner, additional variables were generated by 2 authors: metabolic tumor volume (MTV), $\mathrm{SUV}_{\text {mean }}, \mathrm{SUV}_{\text {peak }}$, and tumor glycolytic volume (TGV) mean and max $\left(\mathrm{TGV}_{\text {max/mean }}\right)$. MTV was measured using a fixed-threshold technique ( $S U V \geq 4)$. $\mathrm{TGV}_{\text {mean }}$ was calculated manually as the product of MTV and $\mathrm{SUV}_{\text {mean }}$ TGVmax was calculated as the product of MTV and $\mathrm{SUV}_{\max }$. mTR was quantified using absolute changes $(\Delta \%)$ and thresholds defined previously (PERCIST and MUNICON criteria; $\mathrm{SUV}_{\max }$ ) (13); additionally, new thresholds were generated by receiver-operator characteristics.

\section{Statistical Analysis}

Analysis was performed using R software (version 3.0.2) (31). Correction for multiple comparisons was performed using the Bonferroni method (32) or false-discovery rate using FDRtoolv1.2.12 (33). For regression, continuous variable distribution was assessed using density plots and transformed $\left(\mathrm{age}^{2} ; \operatorname{logSUV}\right.$ max/mean/peak and time to restaging/surgery). Multivariate analysis included all variables (including PET/CT scanner) after exclusion of perfect separators. Receiver-operator-characteristic optimal thresholds were calculated and compared with pROC (34) (95\% confidence intervals using 200 iterations of 0.632 bootstrapping). Sensitivities and specificities were compared using the McNemar test (DTComPair version 1.0.3) (35).

\section{Model Development, Tuning, Validation, and Performance}

Three techniques were used as previously described (16): logistic regression (backward stepwise binary logistic), decision-tree analysis (recursive partitioning using loss matrices), and artificial neural networks (feed forward back-propagation multilayer perceptron). Models were tuned, generated, and validated internally ( 0.632 bootstrapping) using a development group (patients staged/restaged using the more recent scanner) and validated independently (patients staged/restaged using the earlier scanner; validation group). We partitioned patients in this way to minimize any potential bias, to ascertain immediate clinical utility, and also to assess generalizability to a different scanner system.

\section{RESULTS}

Three hundred two patients underwent resection after NAC. TRG was available for 301 (Tables 1-3). pTR was evident in 82 patients 
TABLE 1

Baseline Patient Characteristics

\begin{tabular}{|c|c|c|c|c|}
\hline Characteristic & Overall $(n=301)$ & $\begin{array}{l}\text { Pathologic } \\
\text { response } \\
(n=82)\end{array}$ & $\begin{array}{l}\text { No pathologic } \\
\text { response } \\
(n=219)\end{array}$ & $\begin{array}{c}P\left(\mathrm{a}=4.55 \times 10^{-3} \text { pre-NAC }\right. \\
\mathrm{a}=2.63 \times 10^{-3} \\
\text { post-NAC) }\end{array}$ \\
\hline Median age $(y)$ & $\begin{array}{c}64.0 \text { (IQR, 58.0-70.0; } \\
\text { range, 36.0-80.0) }\end{array}$ & $\begin{array}{c}62.5 \text { (IQR, 57.3-69.0; } \\
\text { range, 36.0-79.0) }\end{array}$ & $\begin{array}{c}64.0(\mathrm{IQR}, 58.0-70.0 ; \\
\text { range, 38.0-80.0) }\end{array}$ & $0.369^{*}$ \\
\hline Sex & & & & $0.764^{\dagger}$ \\
\hline Male & $228(75.7 \%)$ & $61(74.4 \%)$ & $167(76.3 \%)$ & \\
\hline Female & $73(24.3 \%)$ & $21(25.6 \%)$ & $52(23.7 \%)$ & \\
\hline Cell type & & & & $0.979^{\dagger}$ \\
\hline Adenocarcinoma & $249(82.7 \%)$ & $68(82.9 \%)$ & $181(82.6 \%)$ & \\
\hline Squamous cell carcinoma & $44(14.6 \%)$ & $13(15.9 \%)$ & $31(14.2 \%)$ & \\
\hline Adenosquamous & $5(1.66 \%)$ & $1(1.22 \%)$ & $4(1.83 \%)$ & \\
\hline Small cell carcinoma & $1(0.33 \%)$ & $0(0.00 \%)$ & $1(0.46 \%)$ & \\
\hline Anaplastic & $2(0.66 \%)$ & $0(0.00 \%)$ & $2(0.91 \%)$ & \\
\hline Grade of differentiation & & & & $0.338^{\dagger}$ \\
\hline Well & $28(9.30 \%)$ & $5(6.10 \%)$ & $23(10.5 \%)$ & \\
\hline Moderate & $128(42.5 \%)$ & $35(42.7 \%)$ & $93(42.0 \%)$ & \\
\hline Poor & $140(46.5 \%)$ & $42(51.2 \%)$ & $98(44.7 \%)$ & \\
\hline Undifferentiated & $5(1.66 \%)$ & $0(0.00 \%)$ & $5(2.28 \%)$ & \\
\hline Tumor site & & & & $0.033^{\dagger}$ \\
\hline Mid 1/3 & $18(5.98 \%)$ & $9(11.0 \%)$ & $11(5.02 \%)$ & \\
\hline Distal $1 / 3$ & $52(17.3 \%)$ & $11(13.4 \%)$ & $41(18.7 \%)$ & \\
\hline GEJ 1 & $72(23.9 \%)$ & $23(28.0 \%)$ & $49(22.4 \%)$ & \\
\hline GEJ 2 & 107 (35.5\%) & $20(24.4 \%)$ & $85(38.8 \%)$ & \\
\hline GEJ 3 & 51 (16.9\%) & $19(23.2 \%)$ & $32(14.6 \%)$ & \\
\hline Multifocal & $1(0.33 \%)$ & $0(0.00 \%)$ & $1(0.46 \%)$ & \\
\hline Surgical approach & & & & $0.003^{*}$ \\
\hline LTE & $200(66.4 \%)$ & $12(14.6 \%)$ & $156(71.3 \%)$ & \\
\hline ILE & $46(15.3 \%)$ & $44(53.7 \%)$ & 34 (15.5\%) & \\
\hline 3 stage & $10(3.32 \%)$ & $5(6.10 \%)$ & $5(2.28 \%)$ & \\
\hline THE & $1(0.33 \%)$ & $1(1.22 \%)$ & $0(0.00 \%)$ & \\
\hline ETG & $44(14.6 \%)$ & $20(24.4 \%)$ & $24(11.0 \%)$ & \\
\hline $\begin{array}{l}{ }^{\star} \text { Mann-Whitney test. } \\
{ }^{\dagger} \text { Fisher exact test. } \\
\text { IQR = interquartile range. }\end{array}$ & & & & \\
\hline
\end{tabular}

(27.2\%): TRG 1 in 14 (4.65\%), TRG 2 in 13 (4.32\%), TRG 3 in 55 (18.3\%), TRG 4 in $152(50.5 \%)$, and TRG 5 in $67(22.3 \%)$.

\section{Predicting Pathologic Response Before NAC}

Although there were nominally significant associations between tumor anatomic location and response, on multivariate regression, the only variable that predicted pTR was the use of a triple-agent NAC regimen: odds ratio (OR), $5.98(95 \%$ confidence interval, 2.44-14.7; $P=8.94 \times 10^{-5}$; Table 4).

\section{Predicting Pathologic Response After NAC Using Absolute PET Variables}

A more ${ }^{18} \mathrm{~F}-\mathrm{FDG}$-avid primary tumor after NAC, as quantified by all metrics, was negatively associated with pTR: $\operatorname{logSUV} \mathrm{V}_{\max }$
OR, $3.84 \times 10^{-4}\left(1.17 \times 10^{-5}\right.$ to $2.00 \times 10^{-3} ; P=9.89 \times 10^{-6}$; Table 5; Supplemental Table 1 [supplemental materials are available at http://jnm.snmjournals.org]).

\section{Predicting Pathologic Response Using Metabolic Tumor Response}

mTR predicted pTR (Tables 3 and 6; Supplemental Table 2). This was true both for $\Delta \mathrm{SUV}_{\max }$ and for $\Delta$ length, independently on regression: $\log \Delta \mathrm{SUV}_{\max }$ OR for each percentage reduction, 1.03 (1.01-1.06), $P=$ $3.24 \times 10^{-3} ; \Delta$ length OR, $1.02(1.00-1.03) ; P=0.019$. Interestingly, whereas a PERCIST $\geq 30 \%$ reduction was associated with pTR, the MUNICON $\geq 35 \%$ threshold was not, once adjusted for $\Delta$ length. All additional metrics of mTR were associated with pTR. 
TABLE 2

Pre-NAC Staging Results

\begin{tabular}{|c|c|c|c|c|}
\hline Parameter & $\begin{array}{c}\text { Overall } \\
(n=301)\end{array}$ & $\begin{array}{c}\text { Pathologic } \\
\text { response }(n=82)\end{array}$ & $\begin{array}{l}\text { No pathologic } \\
\text { response }(n=219)\end{array}$ & $\begin{array}{c}P\left(\mathrm{a}=4.55 \times 10^{-3} \text { pre-NAC }\right. \\
\alpha=2.63 \times 10^{-3} \\
\text { post-NAC })\end{array}$ \\
\hline T stage & & & & $0.114^{*}$ \\
\hline 1 & $7(2.33 \%)$ & $2(2.43 \%)$ & $5(2.28 \%)$ & \\
\hline 2 & $46(15.3 \%)$ & $19(23.2 \%)$ & $27(12.3 \%)$ & \\
\hline 3 & $231(76.7 \%)$ & $56(68.3 \%)$ & $175(79.9 \%)$ & \\
\hline $4 a$ & $17(7.76 \%)$ & $5(6.10 \%)$ & $12(35.48 \%)$ & \\
\hline $4 b$ & $0(0.00 \%)$ & $0(0.00 \%)$ & $0(0.00 \%)$ & \\
\hline $\mathrm{N}$ stage & & & & $0.090^{*}$ \\
\hline 0 & $88(29.3 \%)$ & $58(26.5 \%)$ & $30(36.6 \%)$ & \\
\hline 1 & $213(70.7 \%)$ & $161(73.5 \%)$ & $52(63.4 \%)$ & \\
\hline Initial PET/CT & & & & $0.011^{*}$ \\
\hline${ }^{18} \mathrm{~F}-\mathrm{FDG}$-avid & $290(96.7 \%)$ & 75 (91.5\%) & $215(98.2 \%)$ & \\
\hline${ }^{18} \mathrm{~F}-\mathrm{FDG}-$ negative & $11(3.65 \%)$ & 7 (8.54\%) & $4(1.83 \%)$ & \\
\hline Initial PET/CT scanner & & & & $0.897^{\star}$ \\
\hline 1 & $142(47.7 \%)$ & $38(46.3 \%)$ & $104(47.5 \%)$ & \\
\hline 2 & $159(52.3 \%)$ & $44(55.7 \%)$ & $115(52.5 \%)$ & \\
\hline NA & $0(0.00 \%)$ & & & \\
\hline Restaging PET/CT scanner & & & & $0.739^{*}$ \\
\hline 1 & $62(20.6 \%)$ & $16(19.5 \%)$ & $46(21.0 \%)$ & \\
\hline 2 & $158(52.5 \%)$ & $46(56.1 \%)$ & $112(51.19 \%)$ & \\
\hline CT & $81(26.9 \%)$ & $20(24.4 \%)$ & $61(27.9 \%)$ & \\
\hline $\mathrm{mN}$ stage & & & & $0.371^{*}$ \\
\hline 0 (0 nodes) & $209(69.4 \%)$ & $54(65.9 \%)$ & $155(70.8 \%)$ & \\
\hline 1 (1-2 avid nodes) & 54 (17.9\%) & $14(17.1 \%)$ & $40(18.3 \%)$ & \\
\hline 2 (>2 avid nodes) & 38 (12.6\%) & $14(17.1 \%)$ & $24(11.0 \%)$ & \\
\hline NA & $0(0.00 \%)$ & & & \\
\hline Impassable at EGD? & & & & $0.633^{*}$ \\
\hline No & $278(92.4 \%)$ & 77 (93.9\%) & $201(92.8 \%)$ & \\
\hline Yes & $23(7.60 \%)$ & $5(6.10 \%)$ & $18(8.20 \%)$ & \\
\hline
\end{tabular}

${ }^{*}$ Fisher exact test.

$\mathrm{NA}=$ not applicable; EGD = esophagogastroduodenoscopy.

\section{Predicting Pathologic Response Using mNR}

mNR was associated with pTR using the Fisher exact test (Table 3) but not on multivariate regression (Table 6). Notably, mNR and pTR were discordant in 42 of 220 (19.1\%) patients (Table 7). In 41 cases, there was a nodal complete metabolic response or partial metabolic response without pTR, representing $51.2 \%$ of the 82 patients with ${ }^{18}$ F-FDG-avid nodes before NAC (Table 7).

mTR and mNR were also compared (Table 7) and were found to be discordant in $13(5.90 \%)$ cases overall, representing $15.9 \%$ of patients with ${ }^{18} \mathrm{~F}-\mathrm{FDG}$-avid nodes before NAC. Typically, discordance arose due to a mNR in the absence of mTR (10 cases; $4.6 \%$ and $12.2 \%$, respectively).

\section{Defining Optimal Metabolic Response Thresholds}

The accuracy of each continuous (nonthreshold) metric of mTR in predicting pTR is shown in Supplemental Table 3: all were moderately discriminant $(80.2 \%-84.4 \%)$, with no statistically significant differences.

The optimal thresholds for each metric of mTR were determined (Supplemental Table 3), for discrimination, sensitivity, and specificity. The optimal $\Delta \mathrm{SUV}_{\max }$ for sensitivity was a $27.4 \%-30.6 \%$ reduction, identical to PERCIST (30\%) and similar to the MUNICON threshold (35\%). However, specificity was minimal: 33.0\% (23.842.6) and 41.8\% (32.0-52.2), respectively. By contrast, the optimal $\Delta \mathrm{SUV}_{\max }$ threshold for balancing sensitivity (73.6\% [58.6-82.7]) and specificity $(84.5 \%$ [78.7-89.1]) was dramatically different: a $77.8 \%$ reduction. Rounded down to a more pragmatic $75.0 \%$, sensitivity was identical, whereas specificity reduced slightly to $84.0 \%$.

The ability of each mTR metric to predict pTR is shown in Supplemental Tables 4-6. Overall, a $\Delta \mathrm{SUV}_{\max }$ of $77.8 \%$ was significantly more discriminant, with a higher negative predictive value than the PERCIST (30\%) and MUNICON (35\%) thresholds. The same was true for $\Delta \mathrm{MTV}, \Delta \mathrm{TGV}_{\max }$, and $\Delta \mathrm{TGV}_{\text {mean }}$. The highest 
TABLE 3

Surgical Approach and Response to Chemotherapy

\begin{tabular}{|c|c|c|c|c|}
\hline Parameter & Overall $(n=301)$ & $\begin{array}{l}\text { Pathologic } \\
\text { response } \\
(n=82)\end{array}$ & $\begin{array}{l}\text { No pathologic } \\
\text { response } \\
(n=219)\end{array}$ & $\begin{array}{c}P\left(\alpha=4.55 \times 10^{-3}\right. \\
\text { pre-NAC, } \alpha=2.63 \times 10^{-3} \\
\text { post-NAC) }\end{array}$ \\
\hline \multicolumn{5}{|l|}{ Response to chemotherapy } \\
\hline Regimen & & & & $2.69 \times 10^{-5 \star}$ \\
\hline Dual & $230(76.4 \%)$ & $48(58.5 \%)$ & $182(83.1 \%)$ & \\
\hline Triple & $71(23.6 \%)$ & $34(41.5 \%)$ & $37(16.9 \%)$ & \\
\hline $\begin{array}{l}\text { Median days to restaging } \\
\text { scan }\end{array}$ & 82.0 (IQR, 71.0-93.0) & $\begin{array}{c}88.5(\mathrm{IQR}, 71.3-106.8 ; \\
\text { range, 43.0-167) }\end{array}$ & $\begin{array}{l}82.0(\mathrm{IQR}, 71.0-91.0 \\
\text { range, } 40.0-165)\end{array}$ & $0.036^{*}$ \\
\hline $\begin{array}{l}\text { Median days from scan } \\
\text { to surgery }\end{array}$ & $24.0(17.0-33.0)$ & $\begin{array}{l}23.0(\mathrm{IQR}, 18.3-31.8 ; \\
\text { range, 5.0-52.0) }\end{array}$ & $\begin{array}{l}23.0(\mathrm{IQR}, 15.0-33 ; \\
\text { range, 4.0-72.0) }\end{array}$ & $0.283^{*}$ \\
\hline pTR & & NA & NA & NA \\
\hline No & $82(27.2 \%)$ & & & \\
\hline Yes & $219(72.8 \%)$ & & & \\
\hline $\mathrm{mTR}$ & & & & $5.38 \times 10^{-13 *}$ \\
\hline Nonavid & $7(2.33 \%)$ & $5(8.06 \%)$ & $2(1.27 \%)$ & \\
\hline CMR & $48(15.9 \%)$ & $33(53.3 \%)$ & $15(9.49 \%)$ & \\
\hline PMR & 108 (35.9\%) & $20(32.4 \%)$ & $88(55.7 \%)$ & \\
\hline SMD & 43 (14.3\%) & $4(1.33 \%)$ & $39(24.7 \%)$ & \\
\hline PMD & $14(4.65 \%)$ & $0(0.00 \%)$ & $14(8.86 \%)$ & \\
\hline NA & $81(26.9 \%)$ & 20 (NA) & $61(\mathrm{NA})$ & \\
\hline $\mathrm{mNR}$ & & & & $1.23 \times 10^{-4 *}$ \\
\hline No avid nodes & $138(45.8 \%)$ & 39 (62.9\%) & $99(62.6 \%)$ & \\
\hline CMR & $50(16.6 \%)$ & $21(33.9 \%)$ & $29(18.4 \%)$ & \\
\hline PMR/SMD/PMD & $32(10.6 \%)$ & $2(3.22 \%)$ & $30(19.0 \%)$ & \\
\hline NA & $81(26.9 \%)$ & 20 (NA) & 61 (NA) & \\
\hline
\end{tabular}

${ }^{*}$ Fisher exact test.

LTE = left thoracoabdominal esophagectomy; ILE = Ivor-Lewis esophagectomy; THE = transhiatal esophagectomy; ETG = extended total gastrectomy; IQR = interquartile range; $\mathrm{pTR}=$ pathologic tumor response; $\mathrm{mTR}=$ metabolic tumor response; CMR $=$ complete metabolic response; $\mathrm{PMR}=$ partial metabolic response; $\mathrm{SMD}=$ stable metabolic disease; $\mathrm{PMD}=$ progressive metabolic disease; $\mathrm{NA}=$ not applicable.

sensitivities were seen with PERCIST (sensitivity, 100\%), MUNICON (97.1\%), $\Delta \mathrm{MTV}(97.1 \%), \Delta \mathrm{TGV}_{\max }(97.1 \%)$, and $\Delta \mathrm{TGV}_{\text {mean }}$ $(94.3 \%)$; these were significantly more sensitive than $\Delta$ length $\left(<4.68 \times 10^{-3}\right.$; false-discovery rate $\left.=0.046\right)$ but not a $\Delta \mathrm{SUV}_{\max }$ of $77.8 \%$. The most specific were a $\Delta \mathrm{SUV}_{\max }$ of $77.8 \%(81.7 \%$ specific) and $\Delta$ length of $53.1 \%(82.7 \%)\left(P<4.11 \times 10^{-4}\right)$.

\section{Performance of Predictive Models}

Models were generated (Supplemental Table 7) using metrics of $\mathrm{mTR} / \mathrm{mNR}$. The most successful was a logistic regression model comprising $\Delta$ length $+\Delta \mathrm{SUV}_{\text {max }}$; this was highly sensitive $(91.4 \%)$, moderately specific $(71.4 \%)$ and discriminant $(0.814)$, and this sensitivity persisted during internal and independent validation (although with relatively poor specificity and discrimination). However, ultimately none of the composite models outperformed individual mTR thresholds (Supplemental Tables 4-7).

\section{DISCUSSION}

In this study of 301 patients treated with NAC and surgery-the largest to date in esophageal cancer-we found no baseline clinical, tumor, or PET variables associated with pTR. This is perhaps unsurprising, reflecting the daunting complexity involved. Chemoresistance is usually multifactorial and constitutes a spectrum of sensitivity, which depends on numerous macroscopic, microscopic, and molecular factors modulating chemotoxicity $(36,37)$. Intratumoral heterogeneity further complicates chemoresistance, with several subclones, potentially demonstrating differential response and baseline characteristics, in addition to heterogeneity between tumor and nodal metastases. In contrast, after NAC, several PET variables, including absolute tumor metrics and those assessing either mTR or mNR, were strongly associated with pTR on multivariate analysis, and several clinically relevant implications were identified.

First, the identification of a significantly better $\Delta \mathrm{SUV}_{\max }$ threshold (77.8\% reduction) than the generic PERCIST threshold (30\%) suggests that the latter should be raised considerably for esophageal cancer to improve stratification of mTR (perhaps to a more pragmatic 75\%). This threshold was nominally significantly better than the MUNICON threshold (35\%), but as this threshold was originally derived during therapy rather than after therapy (as in our study), the significance of this is uncertain and we are unable to draw further conclusions. 
TABLE 4

Baseline Factors Associated with Pathologic Response to NAC: Univariate and Multivariate Regression

\begin{tabular}{|c|c|c|c|c|}
\hline \multirow[b]{2}{*}{ Factor } & \multicolumn{4}{|c|}{ Response } \\
\hline & Univariate OR (95\% Cl) & $P$ & Multivariate OR $(95 \% \mathrm{Cl})$ & $P$ \\
\hline Median age $(y)$ & $1.00(1.00-1.00)$ & 0.536 & $1.00(1.00-1.00)$ & 0.949 \\
\hline \multicolumn{5}{|l|}{ Sex } \\
\hline Female & Ref & Ref & Ref & Ref \\
\hline Male & $0.90(0.50-1.62)$ & 0.722 & $0.94(0.45-1.95)$ & 0.859 \\
\hline \multicolumn{5}{|l|}{ Cell type } \\
\hline Adenocarcinoma & Ref & Ref & Ref & Ref \\
\hline Squamous cell carcinoma & $1.14(0.56-2.32)$ & 0.716 & $0.87(0.31-2.45)$ & 0.792 \\
\hline \multicolumn{5}{|l|}{ Grade } \\
\hline Well & Ref & Ref & Ref & Ref \\
\hline Moderate & $1.77(0.62-5.02)$ & 0.284 & $1.07(0.33-3.49)$ & 0.906 \\
\hline Poor & $1.99(0.71-5.58)$ & 0.194 & $1.53(0.47-4.97)$ & 0.477 \\
\hline \multicolumn{5}{|l|}{ Site } \\
\hline Mid 1/3 & Ref & Ref & Ref & Ref \\
\hline Distal $1 / 3$ & $0.30(0.10-0.91)$ & 0.034 & $0.21(0.05-0.79)$ & 0.021 \\
\hline GEJ 1 & $0.51(0.18-1.54)$ & 0.200 & $0.34(0.09-1.26)$ & 0.106 \\
\hline GEJ 2 & $0.28(0.10-0.78)$ & 0.015 & $0.17(0.04-0.69)$ & 0.013 \\
\hline GEJ 3 & $0.68(0.23-1.98)$ & 0.480 & $0.15(0.03-0.64)$ & 0.020 \\
\hline \multicolumn{5}{|l|}{ T stage } \\
\hline 1 & Ref & Ref & Ref & Ref \\
\hline 2 & $1.76(0.31-10.0)$ & 0.525 & $2.33(0.34-16.0)$ & 0.390 \\
\hline 3 & $0.83(0.16-4.42)$ & 0.830 & $0.98(0.15-6.27)$ & 0.986 \\
\hline $4 a$ & $1.05(0.15-7.27)$ & 0.967 & $1.13(0.13-10.0)$ & 0.916 \\
\hline \multicolumn{5}{|l|}{$\mathrm{N}$ stage } \\
\hline 0 & Ref & Ref & Ref & Ref \\
\hline 1 & $0.64(0.37-1.10)$ & 0.105 & $0.60(0.13-1.16)$ & 0.129 \\
\hline \multicolumn{5}{|l|}{ Passable at EGD? } \\
\hline Yes & Ref & Ref & Ref & Ref \\
\hline No & $0.63(0.20-1.93)$ & 0.416 & $0.50(0.13-1.95)$ & 0.317 \\
\hline \multicolumn{5}{|l|}{ Chemotherapy } \\
\hline \multicolumn{5}{|l|}{ Regimen } \\
\hline Dual & Ref & Ref & Ref & Ref \\
\hline Triple & $3.48(1.97-6.14)$ & $1.76 \times 10^{-5}$ & $5.98(2.44-14.7)$ & $8.94 \times 10^{-5}$ \\
\hline Log time to restaging & $63.9(4.24-964)$ & $2.66 \times 10^{-3}$ & $10.8(0.42-280)$ & 0.152 \\
\hline Log time to surgery & $0.93(0.31-2.79)$ & 0.896 & $1.12(0.29-4.33)$ & 0.873 \\
\hline \multicolumn{5}{|l|}{$\mathrm{PET} / \mathrm{CT}$ variables } \\
\hline \multicolumn{5}{|l|}{ PET scanner } \\
\hline 1 & Ref & Ref & Ref & Ref \\
\hline 2 & $1.07(0.64-1.79)$ & $(0.796)$ & $0.69(0.36-1.32)$ & 0.267 \\
\hline \multicolumn{5}{|l|}{$\mathrm{mN}$ stage } \\
\hline 0 & Ref & Ref & Ref & Ref \\
\hline 1 & $0.94(0.46-1.89)$ & 0.857 & $1.42(0.60-3.34)$ & 0.426 \\
\hline 2 & $1.67(0.80-3.48)$ & 0.720 & $1.72(0.67-4.45)$ & 0.261 \\
\hline Log SUV $_{\max }$ & $0.43(0.16-1.11)$ & 0.081 & $0.54(0.15-1.92)$ & 0.343 \\
\hline Log ${ }^{18} \mathrm{~F}-\mathrm{FDG}$-avid length & $0.90(0.81-1.01)$ & 0.070 & $0.89(0.77-1.04)$ & 0.145 \\
\hline \multicolumn{5}{|l|}{ Subset of patients ${ }^{*}$} \\
\hline $\mathrm{SUV}_{\text {mean }}$ & $1.47(0.09-23.4)$ & 0.784 & $1.56(0.04-65.8)$ & 0.814 \\
\hline SUV $_{\text {peak }}$ & $2.53(0.41-15.8)$ & 0.320 & $1.85(0.50-6.77)$ & 0.356 \\
\hline MTV & $1.55(0.79-3.04)$ & 0.203 & $1.70(0.66-4.39)$ & 0.276 \\
\hline $\mathrm{TGV}_{\max }$ & $1.53(0.84-2.76)$ & 0.163 & $1.72(0.74-3.99)$ & 0.230 \\
\hline $\mathrm{TGV}_{\text {mean }}$ & $1.45(0.86-2.44)$ & 0.164 & $1.64(0.78-3.42)$ & 0.189 \\
\hline
\end{tabular}

${ }^{*}$ This subset of patients was staged using second PET/CT scanner $(n=155)$.

$\mathrm{Cl}=$ confidence interval; Ref = reference; $\mathrm{NA}=$ not applicable; EGD = esophagogastroduodenoscopy.

Data in parentheses are IQRs. 
TABLE 5

Postchemotherapy Factors Associated with Pathologic Response to NAC: Univariate and Multivariate Regression, Adjusted for Baseline Variables

\begin{tabular}{|c|c|c|c|c|}
\hline \multirow[b]{2}{*}{ Factor } & \multicolumn{4}{|c|}{ Response } \\
\hline & Univariate OR (95\% Cl) & $P$ & Multivariate OR $(95 \% \mathrm{CI})$ & $P$ \\
\hline \multicolumn{5}{|l|}{ Chemotherapy } \\
\hline \multicolumn{5}{|l|}{ Regimen } \\
\hline Dual & Ref & Ref & Ref & Ref \\
\hline Triple & $4.30(2.16-8.55)$ & $3.23 \times 10^{-5}$ & $17.6(4.39-70.1)$ & $5.00 \times 10^{-5}$ \\
\hline Log time to restaging & $25.1(1.10-574)$ & 0.044 & $0.32(0.00-69.2)$ & 0.678 \\
\hline Log time to surgery & $2.28(0.57-9.07)$ & 0.241 & $0.52(0.06-4.82)$ & 0.567 \\
\hline \multicolumn{5}{|l|}{$\mathrm{PET} / \mathrm{CT}$ variables } \\
\hline \multicolumn{5}{|l|}{ PET scanner } \\
\hline 1 & Ref & Ref & Ref & Ref \\
\hline 2 & 1.09 (0.58-2.07) & 0.782 & $0.10(0.02-0.55)$ & 0.008 \\
\hline \multicolumn{5}{|l|}{ Restaging PET scanner } \\
\hline 1 & Ref & Ref & Ref & Ref \\
\hline 2 & $1.30(0.66-2.57)$ & 0.446 & $5.24(0.95-28.9)$ & 0.057 \\
\hline \multicolumn{5}{|l|}{ Restaging $\mathrm{mN}$ stage } \\
\hline 0 (0 avid nodes) & Ref & Ref & Ref & Ref \\
\hline 1 (1-2 avid nodes) & $0.16(0.02-1.28)$ & 0.084 & $1.07(0.07-16.8)$ & 0.959 \\
\hline 2 (>2 avid nodes) & $0.16(0.02-1.28)$ & 0.084 & $2.39(0.18-31.6)$ & 0.509 \\
\hline Restaging log $S U V_{\max }$ & $\begin{array}{c}2.37 \times 10^{-3}\left(4.21 \times 10^{-4}\right. \\
\text { to } 0.01)\end{array}$ & $6.93 \times 10^{-12}$ & $\begin{array}{c}3.84 \times 10^{-4}\left(1.17 \times 10^{-5}\right. \\
\text { to } 0.02)\end{array}$ & $9.89 \times 10^{-6}$ \\
\hline Restaging log avid length & $0.61(0.51-0.73)$ & $3.80 \times 10^{-8}$ & $1.01(0.76-1.34)$ & 0.951 \\
\hline Restaging log MTL & $0.03(0.01-0.10)$ & $3.88 \times 10^{-10}$ & $0.02\left(4.03 \times 10^{-3}\right.$ to 0.06$)$ & $6.19 \times 10^{-9}$ \\
\hline \multicolumn{5}{|c|}{$\begin{array}{l}\text { Subset of patients with } \\
{ }^{18} \mathrm{~F}-\mathrm{FDG} \text {-avid nodes }(n=30)\end{array}$} \\
\hline Log nodal SUV $\max$ & $8.71(0.01-5787)$ & 0.514 & NA & NA \\
\hline \multicolumn{5}{|l|}{$\begin{array}{l}\text { Subset of patients staged } \\
\text { using second PET/CT } \\
\text { scanner }(n=155)\end{array}$} \\
\hline Log SUV & $\begin{array}{c}1.58 \times 10^{-4}\left(7.51 \times 10^{-6}\right. \\
\left.\text { to } 3.23 \times 10^{-3}\right)\end{array}$ & $1.78 \times 10^{-4}$ & $\begin{array}{c}1.13 \times 10^{-7}\left(8.55 \times 10^{-12}\right. \\
\left.\text { to } 1.46 \times 10^{-3}\right)\end{array}$ & $9.32 \times 10^{-5}$ \\
\hline$S U V_{\text {peak }}$ & $\begin{array}{c}5.05 \times 10^{-3}\left(1.81 \times 10^{-4}\right. \\
\text { to } 0.14)\end{array}$ & $1.85 \times 10^{-3}$ & $0.57(0.39-0.84)$ & $3.90 \times 10^{-3}$ \\
\hline Log MTV & $0.28(0.18-0.44)$ & 0.203 & $0.09(0.03-0.28)$ & $2.03 \times 10^{-5}$ \\
\hline $\log \mathrm{TGV}_{\max }$ & $0.32(0.21-0.48)$ & $3.91 \times 10^{-8}$ & $0.11(0.04-0.31)$ & $2.72 \times 10^{-5}$ \\
\hline Log $\mathrm{TGV}_{\text {mean }}$ & $0.30(0.19-0.46)$ & $3.27 \times 10^{-8}$ & $0.10(0.03-0.29)$ & $2.29 \times 10^{-5}$ \\
\hline
\end{tabular}

Second, rather than considering avidity in isolation, we found evidence that incorporating spatial data improved prediction: $\Delta$ length at a most basic level, or ideally a composite metric such as $\triangle \mathrm{MTV}$ or $\Delta \mathrm{TGV}_{\text {max/mean }}$. These outperformed the existing recommended PERCIST threshold of a $30 \% \mathrm{SUV}_{\max }$ reduction. They were comparably sensitive, but more specific $(P<4.11 \times$ $\left.10^{-4}\right)$ and discriminant $\left(P<9.38 \times 10^{-5}\right)$, and were supported by internal (bootstrapping) validation. This suggests that composite metrics may have greater predictive ability in clinical trials than $\triangle \mathrm{SUV}_{\max }$ alone (such as in the MUNICON trial $35 \%$ threshold). In particular, their superior specificity and high negative predictive value (98.5\%-100\%) might identify more nonresponders suitable for cessation of therapy. These findings are in keeping with those of recent smaller studies in chemoradiotherapy; in 20 patients using support vectors and logistic regression, Zhang et al. found mTR quantified using spatial avidity metrics outperformed avidity alone in predicting pTR (38); whereas in 37 patients Jayachandran et al. found MTV to outperform $\mathrm{SUV}_{\max }(39)$.

Third, to our knowledge, this is the first study to assess the novel concept of mNR in association with pTR. We found that the primary tumor and nodal disease often demonstrated a 
TABLE 6

Metabolic Response and Other Factors Associated with Pathologic Response to NAC: Univariate and Multivariate Regression (Patients Staged and Restaged Using Same PET Scanner), Adjusted for

Baseline Variables

\begin{tabular}{|c|c|c|c|c|}
\hline \multirow[b]{2}{*}{ Factor } & \multicolumn{4}{|c|}{ Response } \\
\hline & Univariate OR (95\% Cl) & $P$ & Multivariate OR $(95 \% \mathrm{Cl})$ & $P$ \\
\hline \multicolumn{5}{|l|}{ Chemotherapy } \\
\hline \multicolumn{5}{|l|}{ Regimen } \\
\hline Dual & Ref & Ref & Ref & Ref \\
\hline Triple & $4.30(2.16-8.55)$ & $3.23 \times 10^{-5}$ & $20.3(4.50-91.4)$ & $8.84 \times 10^{-5}$ \\
\hline Log time to restaging & $69.1(1.86-2571)$ & 0.022 & $0.22(0.00-172)$ & 0.658 \\
\hline Log time to surgery & $1.75(0.41-7.44)$ & 0.452 & $0.70(0.06-8.36)$ & 0.781 \\
\hline \multicolumn{5}{|l|}{$\mathrm{PET} / \mathrm{CT}$ variables } \\
\hline \multicolumn{5}{|l|}{ Initial/restaging PET scanner } \\
\hline 1 & Ref & Ref & Ref & Ref \\
\hline 2 & $0.87(0.40-1.88)$ & 0.718 & $0.71(0.21-2.38)$ & 0.580 \\
\hline \multicolumn{5}{|l|}{ nMR } \\
\hline Negative & Ref & Ref & Ref & Ref \\
\hline CMR & $1.93(0.93-4.01)$ & 0.076 & $2.01(0.54-7.51)$ & 0.300 \\
\hline PMR & $0.45(0.05-3.87)$ & 0.465 & $11.2(0.64-197.3)$ & 0.098 \\
\hline SMD & $0.27(0.03-2.18)$ & 0.219 & $1.15(0.09-14.4)$ & 0.911 \\
\hline PMD & NA (NA) & NA & NA (NA) & NA \\
\hline Reduction logSUV $\max (\%)$ & $1.04(1.02-1.05)$ & $6.65 \times 10^{-8}$ & $1.03(1.01-1.06)$ & $3.24 \times 10^{-3}$ \\
\hline Reduction avid length (\%) & $1.03(1.02-1.04)$ & $9.37 \times 10^{-8}$ & $1.02(1.00-1.03)$ & 0.019 \\
\hline \multicolumn{5}{|c|}{ Additional metrics in all patients $(n=202)$} \\
\hline Reduction MTL (\%) & $1.05(1.03-1.07)$ & $2.86 \times 10^{-6}$ & $1.11(1.05-1.16)$ & $1.16 \times 10^{-5}$ \\
\hline \multicolumn{5}{|l|}{ PERCIST (30.0\%) } \\
\hline CMR & Ref & Ref & Ref & Ref \\
\hline PMR & $0.10(0.04-0.22)$ & $2.24 \times 10^{-8}$ & $0.08(0.02-0.32)$ & $3.53 \times 10^{-5}$ \\
\hline SMD/PMD & $0.04(0.01-0.14)$ & $2.18 \times 10^{-7}$ & $0.06(0.01-0.49)$ & $8.46 \times 10^{-4}$ \\
\hline \multicolumn{5}{|l|}{ MUNICON (35.0\%) } \\
\hline No response & Ref & Ref & Ref & Ref \\
\hline Response & $5.21(2.08-13.0)$ & $4.22 \times 10^{-5}$ & $1.63(0.41-6.45)$ & 0.484 \\
\hline \multicolumn{5}{|c|}{$\begin{array}{l}\text { Subset of patients staged using second } \\
\text { PET/CT scanner }(n=155)\end{array}$} \\
\hline Reduction SUV mean $(\%)$ & $1.03(1.02-1.04)$ & $2.25 \times 10^{-8}$ & $1.05(1.02-1.09)$ & $1.90 \times 10^{-3}$ \\
\hline Reduction SUV peak $(\%)$ & $1.09(1.03-1.15)$ & $1.91 \times 10^{-5}$ & $1.04(1.02-1.05)$ & $2.20 \times 10^{-3}$ \\
\hline Reduction MTV (\%) & $1.44(1.09-1.92)$ & $2.70 \times 10^{-5}$ & $1.16(1.07-1.25)$ & 0.011 \\
\hline Reduction TGV $\max (\%)$ & $1.30(1.12-1.52)$ & $5.82 \times 10^{-3}$ & $2.31(1.27-4.20)$ & $2.72 \times 10^{-5}$ \\
\hline Reduction TGV $\mathrm{T}_{\text {mean }}(\%)$ & $1.23(1.10-1.37)$ & $3.91 \times 10^{-8}$ & $1.87(1.20-2.90)$ & $2.29 \times 10^{-5}$ \\
\hline
\end{tabular}

$\mathrm{Cl}=$ confidence interval; Ref = reference; $\mathrm{mNR}=$ metabolic nodal response; $\mathrm{MTL}=$ metabolic tumor length; $\mathrm{CMR}=$ complete metabolic response; $\mathrm{PMR}=$ partial metabolic response; $\mathrm{SMD}=$ stable metabolic disease; $\mathrm{PMD}=$ progressive metabolic disease; $\mathrm{NA}=$ not applicable.

discordant response to NAC, with $\mathrm{mNR}$ seen in the absence of mTR or pTR. With the use of mTR alone (as in the MUNICON trial), this subgroup of patients would be classed as nonresponders and NAC aborted; our findings suggest that in such patients their nodal metastases may in fact be responding to treatment. Nodal metastases by definition contain an aggressive subpopulation of cancer clones originating from the primary tumor, which then evolve differently at a genetic and phenotypic level (40). A crucial such phenotype is chemosensitivity. Although clearly mNR is likely an imperfect surrogate of pathologic nodal response, no systems for assessing nodal response are in use. Our findings are important, as they offer a vital insight into assessing response in the tumor subclones with proven metastatic behavior, likely to be responsible for local and distant disease relapse. 
TABLE 7

Comparison of Tumor and Nodal Metabolic Response

\begin{tabular}{|c|c|c|c|c|c|}
\hline \multirow[b]{2}{*}{ Tumor response } & \multicolumn{5}{|c|}{$\mathrm{mNR}$} \\
\hline & NA & CMR & PMR & SMD & PMD \\
\hline \multicolumn{6}{|l|}{ Pathologic response } \\
\hline pTR & 39 (17.7\%) & $21(9.55 \%)$ & $1(0.45 \%)$ & $1(0.45 \%)$ & $0(0.00 \%$ \\
\hline No pTR & 99 (45.0\%) & $29(13.2 \%)$ & $12(5.45 \%)$ & $13(5.91 \%)$ & $5(22.7 \%)$ \\
\hline \multicolumn{6}{|l|}{ Metabolic response } \\
\hline NA & $6(2.73 \%)$ & $1(0.45 \%)$ & $0(0.00 \%)$ & $0(0.00 \%)$ & $0(0.00 \%)$ \\
\hline CMR & $32(14.5 \%)$ & $14(1.82 \%)$ & $1(0.45 \%)$ & $1(0.45 \%)$ & $0(0.00 \%)$ \\
\hline PMR & 68 (30.9\%) & $29(13.2 \%) 1$ & 8 (3.64\%) & $2(0.91 \%)$ & $0(0.00 \%)$ \\
\hline SMD & 22 (9.09\%) & $5(2.27 \%)$ & $4(1.82 \%)$ & $10(4.55 \%)$ & $3(1.36 \%)$ \\
\hline PMD & $10(4.55 \%)$ & $1(0.45 \%)$ & $0(0.00 \%)$ & $1(0.45 \%)$ & $2(0.91 \%)$ \\
\hline
\end{tabular}

$\mathrm{NA}=$ not applicable; $\mathrm{CMR}=$ complete metabolic response; $\mathrm{PMR}=$ partial metabolic response; $\mathrm{SMD}=$ stable metabolic disease; $\mathrm{PMD}=$ progressive metabolic disease.

This study has several limitations. Although the current gold standard technique for disease response assessment is direct histopathologic examination, this remains imperfect. We used the Mandard classification, which originally described the response of esophageal squamous cell carcinoma to cisplatin-based NACR (27). The Mandard TRG has subsequently been validated for esophageal adenocarcinoma (41) although several other classifications have been described (42); all, however, remain relatively subjective and are tempered by potential interobserver variability and intratumoral sampling bias (43). Ultimately, the Mandard TRG is most frequently used and provides the basis for optimal prediction of survival $(28,30)$. An additional limitation of this study is its retrospective design over a long time period, which although necessary to generate a sufficient cohort resulted in a change of PET/CT scanner, and the availability of additional metrics for the more recent scanner alone. In addition, we included a range of cell types, rather than restricting our analysis. We sought to mitigate these limitations with dedicated review of TRG by a single expert pathologist, by adjusting analyses for cell type and the scanner used and by restricting model development to the more recent representative scanner with subsequent validation in the earlier group, to minimize any bias. We also performed a post hoc analysis comparing metrics between scanners, demonstrating no significant differences in either metabolic response of the primary or nodal tumor $(P=0.109$ [Mann-Whitney] and 0.068 [Fisher exact test]). We believe this to be the largest study performed for esophageal cancer and believe that our results are robust - whether they can be extrapolated to NACR is not clear, but we believe these results warrant urgent assessment. In addition, assessment of several textural response parameters, including entropy and run-length matrices, which although not routinely used in clinical practice have recently been shown to be associated with pTR after NACR (44), and their inclusion in conjunction with volume have been suggested to improve prognostication (45). Such metrics may therefore provide complementary predictive data.

\section{CONCLUSION}

We found that the current definitions used for metabolic response assessment after NAC, based solely on $\Delta \mathrm{SUV}_{\max }$, are both suboptimal and too simplistic and that using composite mea- sures of ${ }^{18}$ F-FDG avidity and volume could significantly improve the predictive ability of PET. The assessment of nodal response, which is often discordant with the primary tumor response, should be urgently studied, as it may offer the potential to further improve response assessment, specifically within tumor populations with proven metastatic behavior.

\section{DISCLOSURE}

John M. Findlay is supported by the NIHR Oxford Biomedical Research Centre. Fergus V. Gleeson is a paid consultant to Alliance Medical. Mark R. Middleton is a paid consultant/ advisor to Amgen, BMS, GSK, Merck, and Millennium and has received institutional funding from Amgen, AZ, BMS, Clovis, Eisai, GSK, Immunocore, Johnson \& Johnson, Merck, Millennium, Novartis, Pfizer, Roche, and Vertex. No other potential conflict of interest relevant to this article was reported.

\section{REFERENCES}

1. Royal College of Surgeons Clinical Effectiveness Unit. National OesophagoGastric Cancer Audit 2012. London, U.K.: Royal College Of Surgeons of England; 2012.

2. Pennathur A, Gibson MK, Jobe BA, Luketich JD. Oesophageal carcinoma. Lancet. 2013;381:400-412

3. Sjoquist KM, Burmeister BH, Smithers BM, et al. Survival after neoadjuvant chemotherapy or chemoradiotherapy for resectable oesophageal carcinoma: an updated meta-analysis. Lancet Oncol. 2011;12:681-692.

4. Urschel JD, Vasan H, Blewett CJ. A meta-analysis of randomized controlled trials that compared neoadjuvant chemotherapy and surgery to surgery alone for resectable esophageal cancer. Am J Surg. 2002;183:274-279.

5. Campbell NP, Villaflor VM. Neoadjuvant treatment of esophageal cancer. World J Gastroenterol. 2010;16:3793-3803.

6. Shapiro J, van Lanschot JJ, Hulshof MC, et al. Neoadjuvant chemoradiotherapy plus surgery versus surgery alone for oesophageal or junctional cancer (CROSS): long-term results of a randomised controlled trial. Lancet Oncol. 2015;16:1090-1098.

7. Kelsen DP, Ginsberg R, Pajak TF, et al. Chemotherapy followed by surgery compared with surgery alone for localized esophageal cancer. $N$ Engl J Med. 1998;339:1979-1984.

8. Findlay JM, Middleton MR, Tomlinson I. A systematic review and meta-analysis of somatic and germline DNA sequence biomarkers of esophageal cancer survival, therapy response and stage. Ann Oncol. 2015;26:624-644. 
9. Hatt M, Visvikis D, Pradier O, Cheze-le Rest C. Baseline ${ }^{18}$ F-FDG PET imagederived parameters for therapy response prediction in oesophageal cancer. Eur J Nucl Med Mol Imaging. 2011;38:1595-1606.

10. Weber WA, Ott K, Becker K, et al. Prediction of response to preoperative chemotherapy in adenocarcinomas of the esophagogastric junction by metabolic imaging. J Clin Oncol. 2001;19:3058-3065.

11. Ott K, Weber WA, Lordick F, et al. Metabolic imaging predicts response, survival, and recurrence in adenocarcinomas of the esophagogastric junction. J Clin Oncol. 2006;24:4692-4698.

12. Lordick F, Ott K, Krause BJ, et al. PET to assess early metabolic response and to guide treatment of adenocarcinoma of the oesophagogastric junction: the MUNICON phase II trial. Lancet Oncol. 2007;8:797-805.

13. Wahl RL, Jacene H, Kasamon Y, Lodge MA. From RECIST to PERCIST: evolving considerations for PET response criteria in solid tumors. J Nucl Med. 2009;50(suppl 1):122S-150S.

14. Davies AR, Gossage JA, Zylstra J, et al. Tumor stage after neoadjuvant chemotherapy determines survival after surgery for adenocarcinoma of the esophagus and esophagogastric junction. J Clin Oncol. 2014;32:2983-2990.

15. Findlay JM, Gillies RS, Franklin JM, et al. Restaging oesophageal cancer after neoadjuvant therapy with F-FDG PET-CT: identifying interval metastases and predicting incurable disease at surgery. Eur Radiol. February 16, 2016 [Epub ahead of print].

16. Findlay JM, Bradley KM, Maile EJ, et al. Pragmatic staging of oesophageal cancer using decision theory involving selective endoscopic ultrasonography, PET and laparoscopy. Br J Surg. 2015;102:1488-1499.

17. Greene FL. The American Joint Committee on Cancer: updating the strategies in cancer staging. Bull Am Coll Surg. 2002;87:13-15.

18. Rice TW, Blackstone EH, Rusch VW. 7th ed. of the AJCC Cancer Staging Manual: esophagus and esophagogastric junction. Ann Surg Oncol 2010;17: 1721-1724.

19. Siewert JR, Stein HJ, Feith M. Adenocarcinoma of the esophago-gastric junction. Scand J Surg. 2006;95:260-269.

20. Medical Research Council Oesophageal Cancer Working Group. Surgical resection with or without preoperative chemotherapy in oesophageal cancer: a randomised controlled trial. Lancet. 2002;359:1727-1733.

21. Phase 2 trial of neo-adjuvant oxaliplatin and 5-fluorouracil in oesophageal cancer. EudraCT 2005-001834-34. EU Clinical Trials Register website. https://www. clinicaltrialsregister.eu/ctr-search/search?query=Adenocarcinoma\&page=17. 2006. Accessed December 28, 2016.

22. A randomised controlled trial comparing standard chemotherapy followed by resection versus ECX chemotherapy followed by resection in patients with resectable adenocarcinoma of the oesophagus. MRC Clinical Trials Unit website. http://www.ctu.mrc.ac.uk/research/documents/cancer_protocols/OE05_Protocol_ Version_6_23_Dec_09.pdf. 2008. Accessed December 28, 2016.

23. A randomised phase II/III trial of perioperative chemotherapy with or without bevacizumab in operable adenocarcinoma of the stomach and gastro-oesophageal junction. MRC Clinical Trials Unit website. http://www.ctu.mrc.ac.uk/our_ research/research_areas/cancer/studies/st03/. 2008. Accessed December 28, 2016.

24. Cunningham D, Allum WH, Stenning SP, et al. Perioperative chemotherapy versus surgery alone for resectable gastroesophageal cancer. $N$ Engl J Med. 2006;355:11-20.

25. Keats AS. The ASA classification of physical status: a recapitulation. Anesthesiology. 1978;49:233-236.

26. Edge SB, Compton CC. The American Joint Committee on Cancer: the 7th ed. of the AJCC cancer staging manual and the future of TNM. Ann Surg Oncol 2010;17:1471-1474.

27. Mandard AM, Dalibard F, Mandard JC, et al. Pathologic assessment of tumor regression after preoperative chemoradiotherapy of esophageal carcinoma: clinicopathologic correlations. Cancer. 1994;73:2680-2686.
28. Chang F, Deere H, Mahadeva U, George S. Histopathologic examination and reporting of esophageal carcinomas following preoperative neoadjuvant therapy: practical guidelines and current issues. Am J Clin Pathol. 2008;129:252-262.

29. Suárez J, Vera R, Balen E, et al. Pathologic response assessed by Mandard grade is a better prognostic factor than down staging for disease-free survival after preoperative radiochemotherapy for advanced rectal cancer. Colorectal Dis. 2008;10:563-568.

30. Karamitopoulou E, Thies S, Zlobec I, et al. Assessment of tumor regression of esophageal adenocarcinomas after neoadjuvant chemotherapy: comparison of 2 commonly used scoring approaches. Am J Surg Pathol. 2014;38:1551-1556.

31. R Core Team. R: A Language and Environment for Statistical Computing. Vienna, Austria: R Foundation for Statistical Computing; 2013.

32. Bland JM, Altman DG. Multiple significance tests: the Bonferroni method. BMJ. 1995;310:170.

33. Klaus BaS. Estimation of (local) false discovery rates and higher criticism. Strimmer Lab website. http://strimmerlab.org/software/fdrtool/. 2014. Accessed December 28, 2016.

34. Robin X, Turck N, Hainard A, et al. pROC: an open-source package for R and $\mathrm{S}+$ to analyze and compare ROC curves. BMC Bioinformatics. 2011;12:77.

35. Stock C, Hielscher, T. DTComPair: comparison of binary diagnostic tests in a paired study design. The Comprehensive R Archive Network website. https:// cran.r-project.org/web/packages/DTComPair/DTComPair.pdf. February 19, 2015. Accessed December 28, 2016.

36. Siddik ZH. Cisplatin: mode of cytotoxic action and molecular basis of resistance. Oncogene. 2003;22:7265-7279.

37. Reardon JT, Vaisman A, Chaney SG, Sancar A. Efficient nucleotide excision repair of cisplatin, oxaliplatin, and Bis-aceto-ammine-dichloro-cyclohexylamine-platinum (IV) (JM216) platinum intrastrand DNA diadducts. Cancer Res. 1999;59:39683971.

38. Zhang H, Tan S, Chen W, et al. Modeling pathologic response of esophageal cancer to chemoradiation therapy using spatial-temporal ${ }^{18}$ F-FDG PET features, clinical parameters, and demographics. Int J Radiat Oncol Biol Phys. 2014;88: 195-203.

39. Jayachandran P, Pai RK, Quon A, et al. Postchemoradiotherapy positron emission tomography predicts pathologic response and survival in patients with esophageal cancer. Int J Radiat Oncol Biol Phys. 2012;84:471-477.

40. Jones S, Chen WD, Parmigiani G, et al. Comparative lesion sequencing provides insights into tumor evolution. Proc Natl Acad Sci USA. 2008;105: 4283-4288.

41. Fareed KR, Al-Attar A, Soomro IN, et al. Tumour regression and ERCC1 nuclear protein expression predict clinical outcome in patients with gastro-oesophageal cancer treated with neoadjuvant chemotherapy. Br J Cancer. 2010;102:16001607.

42. MacGregor TP, Maughan TS, Sharma RA. Pathological grading of regression following neoadjuvant chemoradiation therapy: the clinical need is now. J Clin Pathol. 2012;65:867-871.

43. Chetty R, Gill P, Govender D, et al. A multi-centre pathologist survey on pathological processing and regression grading of colorectal cancer resection specimens treated by neoadjuvant chemoradiation. Virchows Arch. 2012;460: 151-155.

44. Yip SS, Coroller TP, Sanford NN, et al. Relationship between the temporal changes in positron-emission-tomography-imaging-based textural features and pathologic response and survival in esophageal cancer patients. Front Oncol. 2016;6:72.

45. Hatt M, Majdoub M, Vallieres M, et al. ${ }^{18}$ F-FDG PET uptake characterization through texture analysis: investigating the complementary nature of heterogeneity and functional tumor volume in a multi-cancer site patient cohort. $\mathrm{J} \mathrm{Nucl}$ Med. 2015;56:38-44. 